\title{
Rotational Load Flow Method for Radial Distribution Systems
}

\author{
Diego Issicaba* and Jorge Coelho** \\ *Department of Electrical Engineering, Federal University of Technology - Parana (UTFPR), Curitiba-PR, Brazil \\ ** Department of Electrical Engineering, Federal University of Santa Catarina (UFSC), Florianopolis-SC, Brazil
}

\begin{abstract}
Article Info
Article history:

Received Feb 5, 2016

Revised Mar 24, 2016

Accepted Apr 5, 2016

\section{Keyword:}

Power engineering

Power distribution systems

Load flow analysis

Radial networks

ABSTRACT

This paper introduces a modified edition of classical Cespedes' load flow method to radial distribution system analysis. In the developed approach, a distribution network is modeled in different complex reference systems and reduced to a set of connected equivalent subnetworks, each without resistance, while graph topology and node voltage solution are preserved. Active power losses are then not dissipated in the modeled subnetworks and active power flows can be obtained as a consequence of radiality. Thus, the proposed method preprocesses a series of variable transformations concomitant to an iterative algorithm using a forward-backward sweep to arrive at the load flow solution. The proposed approach has been tested using literature and actual distribution networks, and efficiency improvements are verified in comparison to Cespedes' load flow method.
\end{abstract}

Coordinate rotation

Corresponding Author:

Diego Issicaba

Department of Electrical Engineering, Federal University of Technology - Parana (UTFPR)

Av. Sete de Setembro, 3165, Sector D, Rebouas, 80230-910 Curitiba-PR, Brazil

+55 $413310-4626$

issicaba@utfpr.edu.br
Copyright (C) 2016 Institute of Advanced Engineering and Science. All rights reserved.

\section{INTRODUCTION}

Load flow analysis $[1,2]$ provides the steady-state condition for power systems and is one of the most important numerical tools to system planning and designing. In order to design solutions to power distribution system real-time operation [3], modeling and analysis might take into account unbalanced operation and detailed features of each component connected to the networks. On the other hand, in long-term planning stages, some hypotheses and simplifications can be assumed (e.g. balanced operation, constant power load) due to, for instance, uncertainties regarding future load profiles and distributed generation productions. This is the case in adequacy evaluations [4, 5], where hundreds of thousands of load flow analysis may be executed, while performance indices are estimated by modeling failure/repair cycles of components and load/generation profiles as stochastic processes.

Detailed distribution system modeling and analysis is a well established topic, covered in books such as [6]. Simplified/single-phase load flow modeling and analysis dates mostly to the 80's and 90's and can be divided into two groups: the first group comprises Newtonian based methods adjusted to distribution system analysis $[7,8]$ and the second group includes methods based on iterative forward-backward sweep processes $[9,10,11,12,13,14]$. The sweep based techniques are known by their efficiency and take advantage of the fact that distribution networks usually are radially operated.

Forward-backward sweep methods employ the following general procedures: (a) assuming a flat start or an approximate solution, current or power downstream each node is estimated in a backward sweep (from end nodes towards the substation node); (b) using estimates from the previous step, node voltages are updated in a forward sweep (from the substation node towards end nodes); (c) these two steps are repeated up to the convergence of node voltages.

Among the several variations of sweep techniques, the one proposed by Baran and $\mathrm{Wu}[11,12]$ stands out by employing a set of equations, known as Distflow branch equations, that recursively relates active power, reactive power and voltage magnitudes. Cespedes [9] steps ahead by addressing the use of a biquadratic equation to relate node voltages as functions of downstream active and reactive power flows and immediate upstream node voltages.

This paper brings out the fact that, although Cespedes' method is efficient and robust to distribution system analysis, it involves a non-negligible amount of unnecessary calculations associated to updating active and reactive 
power flows in network branches. In fact, a finite and relatively small number of standardized conductor types are utilized in each distribution feeder, composing subnetworks with the same $r+j x \Omega / \mathrm{km}$ and $r / x$ ratio. As consequence, given a possible iterative load flow solution, there is a constant relation between active and reactive losses at each subnetwork branch, making the concomitant effort of updating both, unnecessary.

The proposed approach, of the Rotational Load Flow Method, goes beyond Cespedes' formulation by performing generalized complex reference system rotations and by taking advantage of radiality to improve the efficiency of sweep procedures. The approach has been tested using academic and actual electrical distribution networks, such that efficiency improvements are verified in comparison to Cespedes' load flow method.

The paper is organized into five sections as follows. Section 2 and 3 present mathematical background and the proposed method, respectively. Section 4 shows numerical results for different test systems and an actual system. Conclusions and final remarks are outlined in section 5.

\section{MATHEMATICAL BACKGROUND}

This section presents a brief review of the load flow formulation developed by Cespedes [9]. A discussion about complex reference system rotation, focused on distribution system analysis and applications, is also approached.

\subsection{Cespedes' Load Flow Formulation}

Consider the radial distribution system schematic shown in Fig. 1, where distribution lines are modeled as series impedances $Z_{i}=z_{i} \angle \phi_{i}=r_{i}+j x_{i}$ while complex voltages and complex load demands are represented, respectively, by $V_{i}=v_{i} \angle \theta_{i}$ and $S_{L_{i}}=s_{L_{i}} \angle \delta_{L_{i}}=P_{L_{i}}+j Q_{L_{i}}, \forall i=1, \ldots, N$. Note that the index is utilized to identify the node and the line upstream this node, depending on the variables involved. In addition, $u_{i}$ denotes the node upstream node $i$.

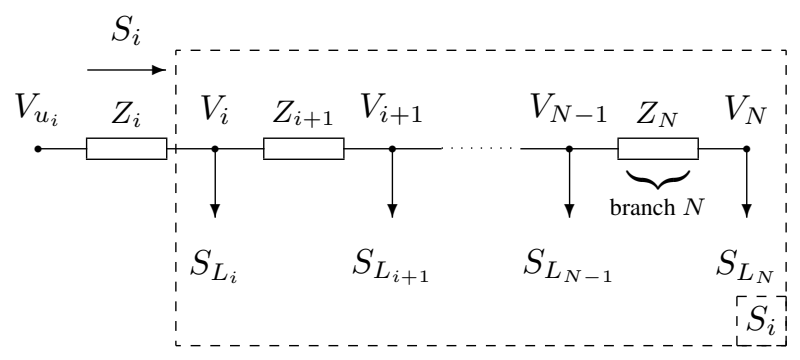

Figure 1. Radial distribution network schematic.

The complex power flow injected at node $i$ through an upstream branch, denoted by $S_{i}=s_{i} \angle \delta_{i}$, can be written as a function of downstream node voltages, loads and line losses. This power flow can be also interpreted as an accumulated power at node $i$ given by

$$
S_{i}=S_{L_{i}}+\sum_{k \in \Delta_{i}} S_{L_{k}}+\sum_{k \in \Delta_{i}} Z_{k}\left(\frac{s_{k}}{v_{k}}\right)^{2}
$$

or, separating in real and imaginary terms,

$$
P_{i}=P_{L_{i}}+\sum_{k \in \Delta_{i}} P_{L_{k}}+\sum_{k \in \Delta_{i}} r_{k}\left(\frac{P_{k}^{2}+Q_{k}^{2}}{v_{k}^{2}}\right), \quad Q_{i}=Q_{L_{i}}+\sum_{k \in \Delta_{i}} Q_{L_{k}}+\sum_{k \in \Delta_{i}} x_{k}\left(\frac{P_{k}^{2}+Q_{k}^{2}}{v_{k}^{2}}\right)
$$

where $\Delta_{i}$ denotes the set of nodes downstream node $i$ and the set of lines downstream node $i$, depending on the variables involved.

Once accumulated active and reactive powers at node $i$ are known, the voltage magnitude at node $i$ can be obtained by solving the biquadratic equation [9]

$$
v_{i}^{4}+A_{i} v_{i}^{2}+B_{i}=0
$$

where

$$
A_{i}=2\left(P_{i} r_{i}+Q_{i} x_{i}\right)-v_{u_{i}}^{2}, \quad B_{i}=\left(P_{i}^{2}+Q_{i}^{2}\right)\left(r_{i}^{2}+x_{i}^{2}\right)
$$

Similarly, the angle at node $i$ can be obtained through the following equation. 


$$
\theta_{i}=\theta_{i-1}+\tan ^{-1}\left(\frac{P_{i} x_{i}-Q_{i} r_{i}}{P_{i} r_{i}+Q_{i} x_{i}+v_{i}^{2}}\right)
$$

Thus, assuming that the substation node complex voltage $V_{0}=v_{0} \angle 0^{\circ}$ is specifed and node voltage magnitudes are initiated using a flat start or an approximate solution, the load flow solution can be found through iterative forward-backward sweeps as follows. In a backward process, accumulated power flow at each node is calculated using (2), starting at end-nodes and stopping at the first node immediately downstream from the substation node. In a forward process, voltage magnitudes are updated, away from the substation node, using (3)-(4). These procedures are continually repeated until the convergence of voltage magnitudes is reached.

The backward process is the most time consuming part of the algorithm and involves the calculation of power losses and power accumulation through network branches. However, a finite and relatively small number of standardized conductor types are installed in a distribution network, each one with a specified $r / x$ ratio. These ratios can be mathematically controlled through a proper reference system rotation, as it will be exposed in the following subsection.

\subsection{Complex Reference System Rotation} in Fig. 2.

Assume an impedance $Z_{i}$ represented in an alternative complex reference system rotated by $\varphi$, as illustrated

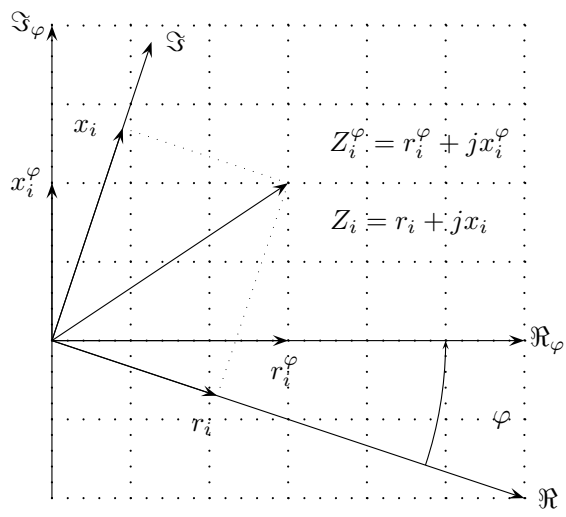

Figure 2. Impedance represented into two different complex reference systems phased by a $\varphi$ angle.

The complex value of the impedance in the alternative reference system can be obtained by

$$
Z_{i}^{\varphi}=Z_{i} e^{j \varphi}=r_{i}^{\varphi}+j x_{i}^{\varphi}
$$

where

$$
r_{i}^{\varphi}=r_{i} \cos \varphi-x_{i} \sin \varphi, \quad x_{i}^{\varphi}=r_{i} \sin \varphi+x_{i} \cos \varphi
$$

and the updated $r / x$ ratio given by

$$
\frac{r_{i}^{\varphi}}{x_{i}^{\varphi}}=\frac{r_{i} \cos \varphi-x_{i} \sin \varphi}{r_{i} \sin \varphi+x_{i} \cos \varphi} \quad \text { for } x_{i}^{\varphi} \neq 0
$$

Analogously, a complex apparent load in the alternative reference system is given by

$$
S_{L_{i}}^{\varphi}=S_{L_{i}} e^{j \varphi}=P_{L_{i}}^{\varphi}+j Q_{L_{i}}^{\varphi}
$$

where

$$
P_{L_{i}}^{\varphi}=P_{L_{i}} \cos \varphi-Q_{L_{i}} \sin \varphi, \quad Q_{L_{i}}^{\varphi}=P_{L_{i}} \sin \varphi+Q_{L_{i}} \cos \varphi
$$

Once all impedances and loads are rotated by (6) and (9), the system model is represented in the alternative reference system. Hence, the complex power injected at node $i$ through an upstream branch is also rotated by $\varphi$, such that

$$
S_{i}^{\varphi}=S_{i} e^{j \varphi}=P_{i}^{\varphi}+j Q_{i}^{\varphi}
$$


where

$$
P_{i}^{\varphi}=P_{i} \cos \varphi-Q_{i} \sin \varphi, \quad Q_{i}^{\varphi}=P_{i} \sin \varphi+Q_{i} \cos \varphi
$$

Since the system model is represented in the alternative reference system, the load flow voltage solution is also rotated by $\varphi$, though voltage magnitudes remain unaltered. In fact, using (3)-(4), the biquadratic voltage equation can be rewritten in the alternative reference system as

$$
\left(v_{i}^{\varphi}\right)^{4}+A_{i}^{\varphi}\left(v_{i}^{\varphi}\right)^{2}+B_{i}^{\varphi}=0
$$

where

$$
A_{i}^{\varphi}=2\left(P_{i}^{\varphi} r_{i}^{\varphi}+Q_{i}^{\varphi} x_{i}^{\varphi}\right)-\left(v_{u_{i}}^{\varphi}\right)^{2}, \quad B_{i}^{\varphi}=\left(\left(P_{i}^{\varphi}\right)^{2}+\left(Q_{i}^{\varphi}\right)^{2}\right)\left(\left(r_{i}^{\varphi}\right)^{2}+\left(x_{i}^{\varphi}\right)^{2}\right)
$$

By substituting (7) and (12) in (14), as well as by assuming $v_{u_{i}}^{\varphi}=v_{u_{i}}$, we obtain that $A_{i}^{\varphi}=A_{i}$ and $B_{i}^{\varphi}=B_{i}$. As consequence $v_{i}^{\varphi}=v_{i}$, causing that voltage magnitude solutions are preserved.

Once the same voltage value is chosen for the substation node in both reference systems, i.e. $v_{0}^{\varphi}=v_{0}$, the condition $v_{u_{i}}^{\varphi}=v_{u_{i}}$ is always satisfied because of the order in which the nodes are visited in the ladder iterative procedures. Therefore, a network represented in the alternative reference system and other represented in the conventional reference system present both the same load flow voltage magnitudes, independently of the rotation angle chosen. In addition, the load flow procedures will present the same voltage iterates in both reference systems.

One important application of reference system rotation is that a distribution network can now be represented in a coordinate system where the $r / x$ ratios assume low figures, improving the Newtonian based load flow algorithms in terms of robustness and efficiency. The proposed approach goes beyond such concept by introducing generalized complex reference system rotations to take advantage of distribution network radiality in Cespedes' ladder iterative formulation.

\section{ROTATIONAL LOAD FLOW METHOD}

This section concerns the proposed approach and is organized for didactic reasons into two cases: a case comprising networks with a single conductor type and a general case with different conductor types installed through distribution networks.

\subsection{Single cable type case}

Consider a network with only one conductor type installed. By hypothesis, complex impedances present the same $r / x$ ratio and $\phi$ angle. As exposed previously, $r / x$ ratios can be controlled by a complex reference system rotation. For the general schematic shown in Fig. 1, by conveniently choosing the rotation angle $\varphi=\frac{\pi}{2}-\phi$ and by Kirchhoff's current law we obtain

$$
V_{u_{i}}-V_{i}=Z_{i}^{\varphi}\left(\frac{S_{i}^{\varphi}}{V_{i}}\right)^{*}=j z_{i}\left(\frac{S_{i}^{\varphi}}{V_{i}}\right)^{*}
$$

Moreover, using (1) and (11), the rotated accumulated complex power at node $i$ can be computed by

$$
S_{i}^{\varphi}=S_{L_{i}} e^{j \varphi}+\sum_{k \in \Delta_{i}} S_{L_{k}} e^{j \varphi}+\sum_{k \in \Delta_{i}} Z_{k} e^{j \varphi}\left(\frac{s_{k}}{v_{k}}\right)^{2}=S_{L_{i}}^{\varphi}+\sum_{k \in \Delta_{i}} S_{L_{k}}^{\varphi}+\sum_{k \in \Delta_{i}} j z_{k}\left(\frac{s_{k}}{v_{k}}\right)^{2}
$$

Separating the real and imaginary terms, and denoting $L_{Q_{i}}^{\varphi}$ as the rotated reactive network losses at branch $i$, we have

$$
\begin{gathered}
P_{i}^{\varphi}=P_{L_{i}}^{\varphi}+\sum_{k \in \Delta_{i}} P_{L_{k}}^{\varphi} \\
Q_{i}^{\varphi}=Q_{L_{i}}^{\varphi}+\sum_{k \in \Delta_{i}} Q_{L_{k}}^{\varphi}+L_{Q_{i}}^{\varphi}
\end{gathered}
$$

where

$$
L_{Q_{i}}^{\varphi}=\sum_{k \in \Delta_{i}} z_{k}\left(\frac{s_{k}}{v_{k}}\right)^{2}
$$

Using the variable transformation, the accumulated active power at node $i$ can be directly obtained summing rotated active load demands downstream from node $u_{i}$. Therefore, there will not be any active losses found and active 
power flow calculation through network branches becomes straightforward. Consequently, load and network data can be stored in a convenient coordinate reference system and the sweep algorithm can now be formulated to concern only the calculation of reactive losses, as described in Fig. 3.

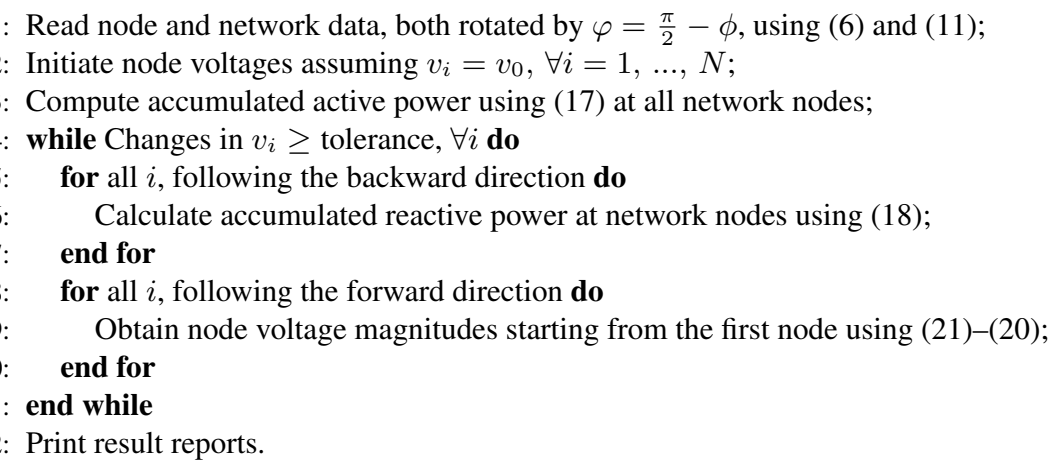

Figure 3. Rotational Load Flow algorithm - Single conductor type case with constant power load modeling.

The biquadratic equation becomes simplified by removing resistances from the formulation, as follows.

$$
v_{i}=\left(\frac{-A_{i}^{\varphi}+\left(\left(A_{i}^{\varphi}\right)^{2}-4 B_{i}^{\varphi}\right)^{1 / 2}}{2}\right)^{1 / 2}
$$

where

$$
\left.A_{i}^{\varphi}=2 x_{i}^{\varphi} Q_{i}^{\varphi}-v_{u_{i}}^{2}, \quad B_{i}^{\varphi}=\left(\left(P_{i}^{\varphi}\right)^{2}+Q_{i}^{\varphi}\right)^{2}\right)\left(x_{i}^{\varphi}\right)^{2}
$$

By eliminating all these calculations, non-negligible CPU time can be saved.

\subsection{General case}

Consider a general distribution network with lateral branches and voltage dependent load demands. Notice that this network can be divided into a set of connected subnetworks with the same conductor type. These subnetworks can be represented in a convenient coordinate system to eliminate the line resistances from modeling, as illustrated in Fig. 4.
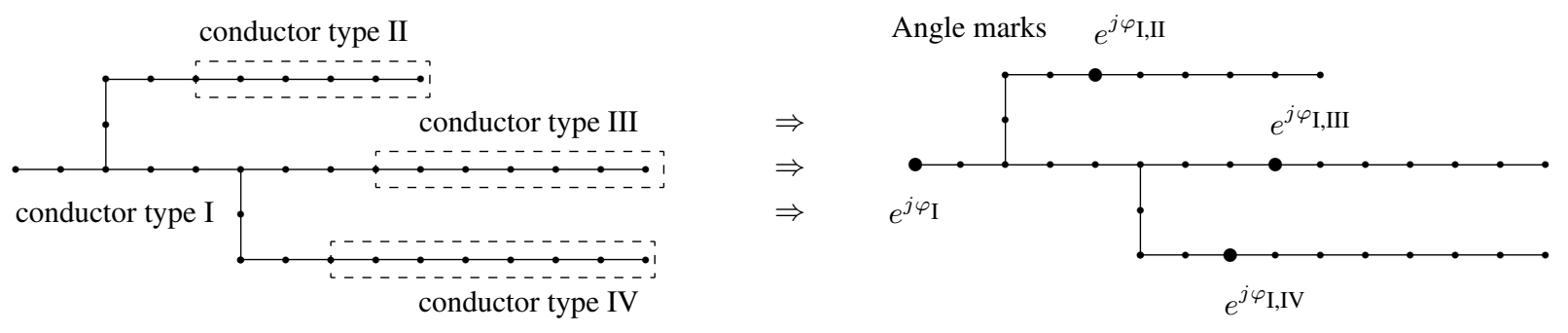

Figure 4. Rotational Load Flow principle - General case.

By preserving the topological structure and load flow voltage solution, the power will flow through the subnetworks assuming different representations for each reference system. Hence, the load flow formulation can be simplified to eliminate the active losses from modeling by mathematically handling the differences of coordinate reference systems among subnetworks. This accomplishment is obtained through a generalized complex reference system rotation algorithm, shown in Fig. 5, in which stored data is changed and connections between subnetworks are assigned by angle marks. The rotation procedures are identical for the case of modeling other series components by series impedances. 


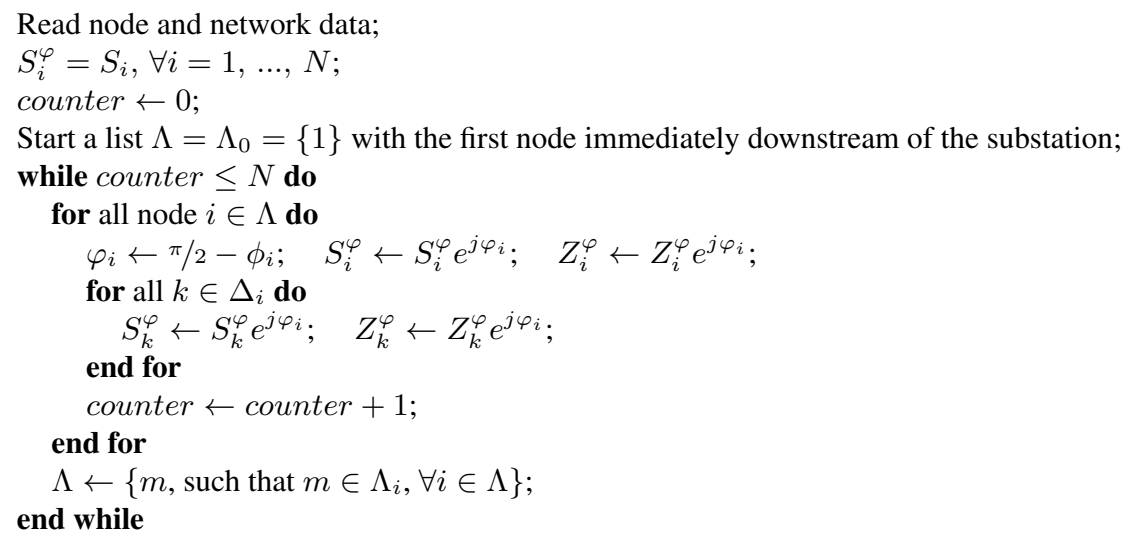

Figure 5. Generalized complex reference system rotation.

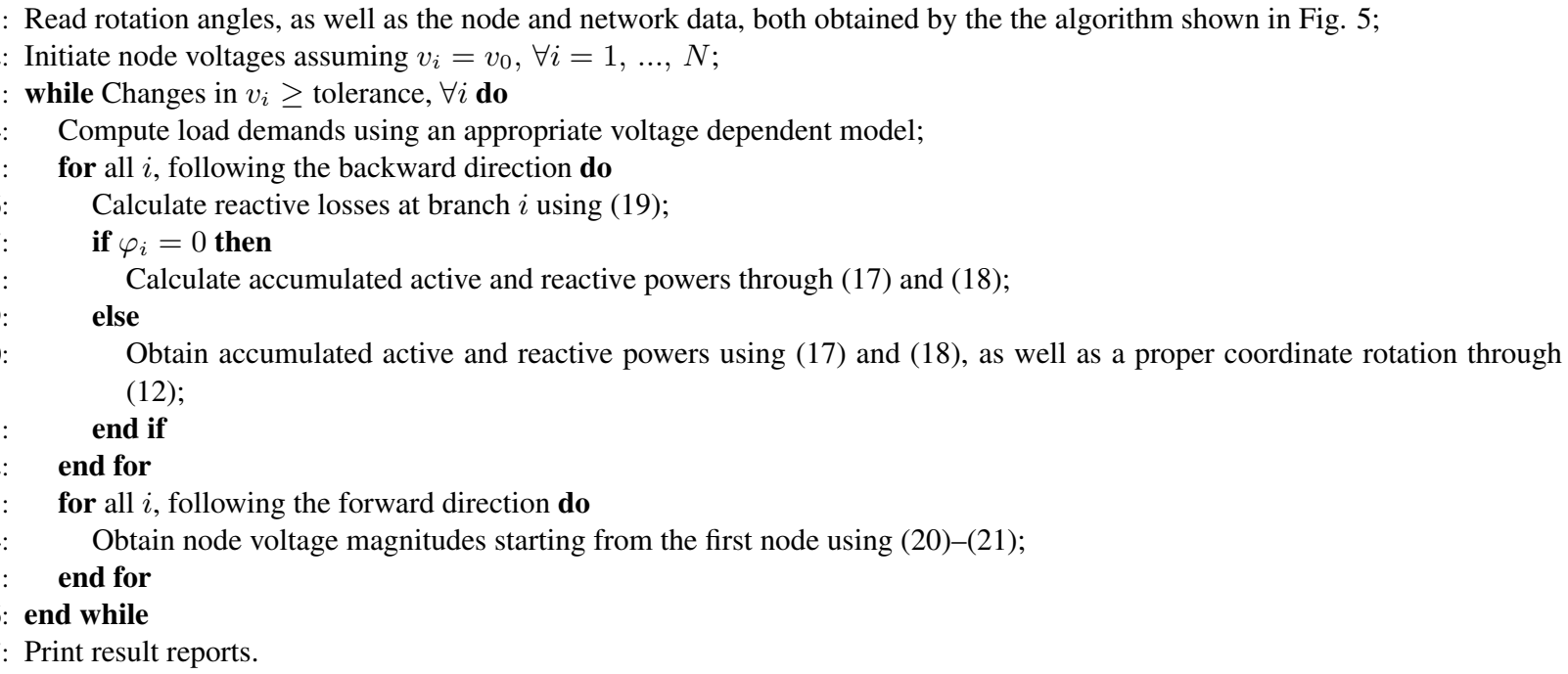

Figure 6. Rotational Load Flow algorithm - General case.

The Rotational Load Flow method for radial distribution networks is presented in Fig. 6. The approach utilizes a sweep algorithm and performs complex reference system rotations of accumulated powers whereas a subnetwork connections is assigned. In the forward procedure, rotations are not required and the basic biquadratic equation is simplified with the removing of line resistances from modeling.

\section{RESULTS AND DISCUSSIONS}

The proposed approach was implemented in MATLAB using an old computer (1,66 GHz, Core Duo), and it has been extensively tested with distribution networks obtained from literature and with actual distribution networks. For all simulations, tolerance was set up in 0.000001 and initial voltage magnitudes were assumed 1 pu. Voltage dependent loads were modeled using the polynomial form

$$
P_{L_{i}}=P_{L_{i}}^{0}\left(\alpha_{P}+\beta_{P} v_{i}+\gamma_{P} v_{i}^{2}\right), \quad Q_{L_{i}}=Q_{L_{i}}^{0}\left(\alpha_{Q}+\beta_{Q} v_{i}+\gamma_{Q} v_{i}^{2}\right)
$$

where $P_{L_{i}}^{0}$ and $Q_{L_{i}}^{0}$ are the rated active and reactive loads, respectively. The coefficients $\left(\alpha_{P}, \beta_{P}, \gamma_{P}\right)$ and $\left(\alpha_{Q}, \beta_{Q}, \gamma_{Q}\right)$ were set in $(0.8,0.1,0.1)$.

Cespedes' method and the Rotational Load Flow approach were applied to solve load flow problems in a 12node network [15], 27-node network [15], 29-node network [9], 32-node network [12] and a 69-node network [11]. 
Table 1 shows simulation results achieved with these comparative case-studies.

Table 1. Simulation results for the rotational and Cespedes' methods using distribution networks obtained in the literature

\begin{tabular}{|c|c|c|c|c|c|c|}
\hline \multirow{2}{*}{ Ref. } & \multirow{2}{*}{$\mathrm{N}$} & \multicolumn{2}{|c|}{ Runtime (ms) } & \multirow{2}{*}{ Iter. } & \multirow[t]{2}{*}{$\tau$} & \multirow{2}{*}{$\begin{array}{c}\text { Saving } \\
\text { (\%) }\end{array}$} \\
\hline & & $\mathrm{CM}$ & RLFM & & & \\
\hline [15] & 11 & 56.9101 & 45.4312 & 4 & 0.1666 & 25.27 \\
\hline [15] & 27 & 58.4912 & 48.7431 & 5 & 0.2222 & 20.01 \\
\hline [12] & 32 & 56.4634 & 47.1671 & 5 & 0.7188 & 19.71 \\
\hline [9] & 29 & 57.9068 & 48.5995 & 6 & 0.9655 & 19.15 \\
\hline [11] & 69 & 61.8391 & 50.1512 & 5 & 0.1884 & 23.31 \\
\hline
\end{tabular}

CM: Cespedes' method.

RLFM: Rotational Load Flow method.

$\tau$ : Ratio between the number of changes on conductor types and the number of network nodes.

The comparative studies indicate that the proposed approach provides superior results in term of efficiency when compared to Cespedes' technique. In fact, calculations of active losses have been eliminated in the proposed approach and sweep procedures were adapted to handle differences of complex reference system representations. Node voltages are equally updated in both methods following that the number of iterations necessary for convergence are also preserved in the proposed approach. Also, it was not found any sacrifice in solution quality by using the rotation technique.

A similar case study was devised for an actual distribution network, shown in Fig. 7. This network is composed of 236 nodes, 1337 kilometers of lines and 5 conductor types installed. Simulation results for this network are presented in Table 2.

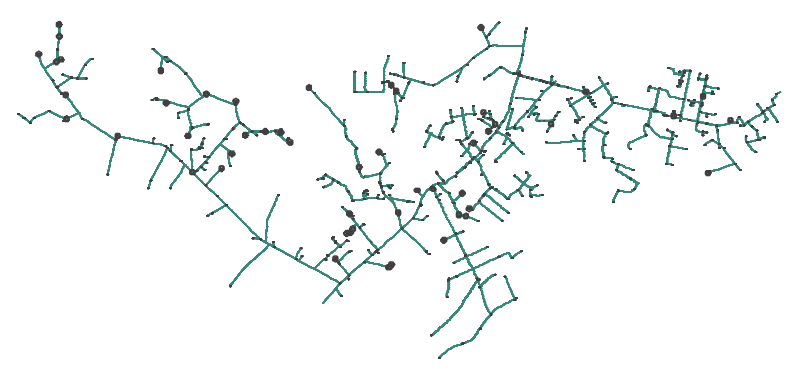

Figure 7. An actual radial structured distribution network.

Table 2. Simulation results for the rotational and Cespedes' methods using an actual distribution network

\begin{tabular}{cccccc}
\hline \multirow{2}{*}{$\mathrm{N}$} & \multicolumn{2}{c}{ Runtime (ms) } & Iter. & $\tau$ & Saving \\
& $\mathrm{CM}$ & RLFM & & & $(\%)$ \\
\hline 236 & 70.0321 & 55.4044 & 5 & 0.1525 & 26.40 \\
\hline
\end{tabular}

CM: Cespedes' method.

RLFM: Rotational Load Flow method.

$\tau$ : Ratio between the number of changes on conductor types and the number of network nodes.

Extensive simulations pointed out runtime improvements depend upon the ratio between the number of changes in conductor types across the network and the number of network nodes. This fact was expected since a large number of changes in conductor type might increase the number of rotations during the backward procedures, as formalized in Appendix A. All outcomes, either using literature or actual networks, indicate that the proposed method provides improved results in comparison to Cespedes' method in term of efficiency. 


\section{CONCLUSIONS AND FINAL REMARKS}

Adequacy evaluations usually require a large number of load flow computations to estimate a small set of performance indices. These evaluations are utilized in long-term planning stages, where simplifications such as balanced operation and constant power load demands can be assumed, while efforts are directed to accurately modeling the failure/repair cycles of network components and load/generation profiles as stochastic processes.

Aiming at achieving improved efficiency in adequacy evaluations, a modified version of Cespedes' load flow method to radial distribution systems is proposed. The approach eliminates unnecessary calculations in Cespedes' load flow method by representing distribution systems in different and conveniently chosen coordinate reference systems. For this accomplishment, a generalized complex reference systems rotation algorithm was introduced and the load flow formulation was adapted to handle differences in coordinate reference system representations.

Result analysis obtained from both literature and actual networks indicate that the proposed method provide improved efficiency in comparison to Cespedes' method. Future works will investigate extensions of the proposed approach with regard to unbalanced distribution networks, dispersed generation, and weakly-meshed distribution systems.

\section{ACKNOWLEDGMENTS}

The authors would like to acknowledge the financial, technical and human support of the CNPq, CAPES and INESC P\&D Brasil.

\section{REFERENCES}

[1] H. Marefatjou and M. Sarvi, "Distributed generation allocation to improve steady state voltage stability of distribution networks using imperialist competitive algorithm," International Journal of Applied Power Engineering (IJAPE), vol. 2, no. 1, pp. 15-26, 2013.

[2] N. Ghaffarzadeh, M. Akbari, and A. Khanjanzadeh, "Distributed generation allocation to improve steady state voltage stability of distribution networks using imperialist competitive algorithm," International Journal of Applied Power Engineering (IJAPE), vol. 2, no. 2, pp. 71-78, 2013.

[3] D. Issicaba, A. J. S. Costa, and J. L. Colombo, "Real-time monitoring of points of common coupling in distribution systems through state estimation and geometric tests," IEEE Transactions on Smart Grid, vol. 7, no. 1, pp. 9-18, Jan. 2016.

[4] D. Issicaba, J. A. P. Lopes, and M. A. Rosa, "Adequacy and security evaluation of distribution systems with distributed generation," IEEE Transactions on Power Systems, vol. 27, no. 3, pp. 1681-1689, Aug. 2012.

[5] D. Issicaba, M. A. Rosa, and J. A. P. Lopes, "Distribution systems performance evaluation considering islanded operation," in Proceedings of the Power System Computation Conference, Stockholm, Sweden, 2011.

[6] W. H. Kersting, Distribution System Modeling and Analysis, 2nd ed., ser. The Electrical Engineering Series, L. Grigsby, Ed. CRC Press, Jul. 2011.

[7] D. Radicic and A. Bose, "A modification to the fast decoupled power flow to networks with high r/x ratios," IEEE Transactions on Power Systems, vol. 3, no. 2, pp. 743-746, May 1988.

[8] F. Zhang and C. S. Cheng, "A modified newton method for radial distribution system power flow analysis," IEEE Transactions on Power Systems, vol. 12, no. 1, pp. 389-397, Feb. 1997.

[9] R. G. Cespedes, "New method for the analysis of distribution networks," IEEE Transactions on Power Delivery, vol. 5, no. 1, pp. 391-396, Jan. 1990.

[10] D. Shirmohammadi, H. W. Hong, A. Semlyen, and G. X. Luo, "A compensation-based power flow method for weakly meshed distribution and transmission networks," IEEE Transactions on Power Systems, vol. 3, no. 2, pp. 753-762, May 1988.

[11] M. E. Baran and F. F. Wu, "Optimal sizing of capacitor placed on a radial distribution system," IEEE Transaction on Power Delivery, vol. 4, no. 1, pp. 735-743, Jan. 1989.

[12] _ _ "Network reconfiguration in distribution systems for loss reduction and load balancing," IEEE Transactions on Power Delivery, vol. 4, no. 2, pp. 1401-1407, Apr. 1989.

[13] M. H. Haque, "Load flow solution of distribution systems with voltage dependent load models," Electric Power Systems Research, vol. 36, no. 3, pp. 151-156, Mar. 1996.

[14] _ _ "Efficient load flow method for distribution systems with radial or mesh configuration," in IEE Proceedings on Generation, Transmission and Distribution, vol. 143, no. 1, Jan. 1996, pp. 33-38.

[15] D. Das, H. S. Nagi, and D. P. Kothari, "Novel method for solving radial distribution networks," in IEE Proceedings on Gen., Tran. and Distr., vol. 141, no. 4, Jul. 1994, pp. 291-298. 


\section{APPENDIX A. RELATION BETWEEN NUMBER OF ROTATIONS AND NUMBER OF NODES}

The proposed approach can be compared to Cespedes' method according to the usage of basic mathematical operations per iteration such as: multiplication/division $(\otimes \cup \oslash)$, sum/subtraction $(\oplus \cup \ominus)$ and square root $(\sqrt{ }$ ). Although the usage of mathematical operations may vary according to actual implementation and the number of operations to rotating system data depends on the system itself, it is possible to relate the operations per iteration of both methods in order to identify whether the proposed approach will be more efficient for a given adequacy study. In our implementation, Cespedes' method utilizes in each iteration the calculations listed below.

$$
\begin{gathered}
I_{i}^{2} \leftarrow \frac{P_{i}^{2}+Q_{i}^{2}}{v_{i}^{2}} ; P_{u_{i}} \leftarrow P_{u_{i}}+P_{i}+r_{i} I_{i}^{2} ; Q_{u_{i}} \leftarrow Q_{u_{i}}+Q_{i}+x_{i} I_{i}^{2} \Rightarrow N \text { times } \\
A_{i} \leftarrow 2\left(r_{i} P_{i}+x_{i} Q_{i}\right)-v_{u_{i}}^{2} ; B_{i} \leftarrow\left(P_{i}^{2}+Q_{i}^{2}\right)\left(r_{i}^{2}+x_{i}^{2}\right) ; v_{i} \leftarrow\left(\frac{-A_{i}+\left(A_{i}^{2}-4 B_{i}\right)^{1 / 2}}{2}\right)^{1 / 2} \Rightarrow N \text { times }
\end{gathered}
$$

totalizing, in terms of number of operations,

$$
18 N(\otimes \cup \oslash)+11 N(\oplus \cup \ominus)+2 N(\sqrt{\cdot})
$$

Similarly, let $M$ be the number of power flow rotations between subnetworks, the basic mathematical operations per iteration of the proposed approach are the following:

$$
\begin{gathered}
L_{Q_{i}} \leftarrow x_{i}\left(\frac{P_{i}^{2}+Q_{i}^{2}}{v_{i}^{2}}\right) \Rightarrow N \text { times } \\
P_{u_{i}} \leftarrow P_{u_{i}}+P_{i} \Rightarrow(N-M) \text { times } \\
Q_{u_{i}} \leftarrow Q_{u_{i}}+Q_{i}+L_{Q_{i}} \Rightarrow(N-M) \text { times } \\
A_{i} \leftarrow 2 x_{i} Q_{i}-v_{u_{i}}^{2} \Rightarrow N \text { times } \\
B_{i} \leftarrow\left(P_{i, a c}^{2}+Q_{i, a c}^{2}\right) x_{i}^{2} \Rightarrow N \text { times } \\
v_{i} \leftarrow\left(\frac{-A_{i}+\left(A_{i}^{2}-4 B_{i}\right)^{1 / 2}}{2}\right)^{1 / 2} \Rightarrow N \text { times } \\
P_{u_{i}} \leftarrow P_{u_{i}}+P_{i} \cos \varphi-\left(Q_{i}+L_{Q_{i}}\right) \sin \varphi \Rightarrow M \text { times } \\
Q_{u_{i}} \leftarrow Q_{u_{i}}+P_{i} \sin \varphi+\left(Q_{i}+L_{Q_{i}}\right) \cos \varphi \Rightarrow M \text { times }
\end{gathered}
$$

totalizing, also in terms of number of operations,

$$
(15 N+4 M)(\otimes \cup \oslash)+(8 N+3 M)(\oplus \cup \ominus)+2 N(\sqrt{ } \cdot)
$$

By using (23) and (24), we conclude that the proposed approach will be advantageous in terms of number of operations per iteration whether

$$
(3 N-4 M)(\otimes \cup \oslash)+(3 N-3 M)(\oplus \cup \ominus)>0
$$

This inequality is true, for instance, if $M<0,75 N$, which is a condition easily satisfied in actual networks.

\section{BIOGRAPHIES OF AUTHORS}

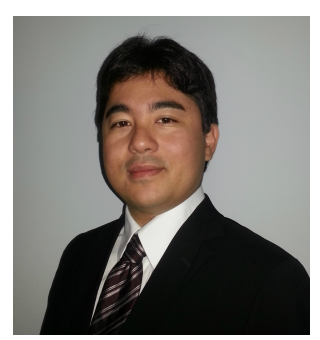

Diego Issicaba received the B.S. and M.S. degrees in Electrical Engineering from the Federal University of Santa Catarina (UFSC), Santa Catarina, Brazil, in 2006 and 2008, respectively. Furthermore, he received the Ph.D. degree on Sustainable Energy Systems, under the MIT Doctoral Program, from the Faculty of Engineering of the University of Porto, Portugal. His research interests involve smart grids, mutiagent systems, distributed generation and distribution systems. He is currently a full Professor at Federal University of Technology - Parana (UTFPR), Associate Researcher and Coordinator of the Research Area on Energy and Management of INESC P\&D Brasil.

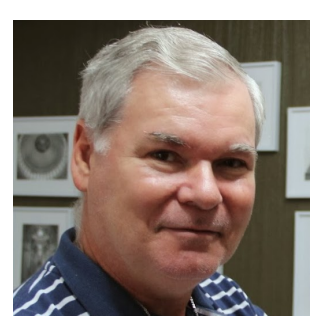

Jorge Coelho received the B.S. and M.S. degrees in electrical engineering from the Federal University of Santa Catarina, Brazil, in 1977 and 1980, respectively. In 1990, he received the Ph.D. degree in electrical engineering from the Catholic University of Rio de Janeiro, Brazil. He is a Professor of the Department of Electrical Engineering at the Federal University of Santa Catarina, Brazil, since March 1978. His research interests include distribution systems expansion and operation planning, power systems reliability, probabilistic methods applied to power systems, and power quality. 